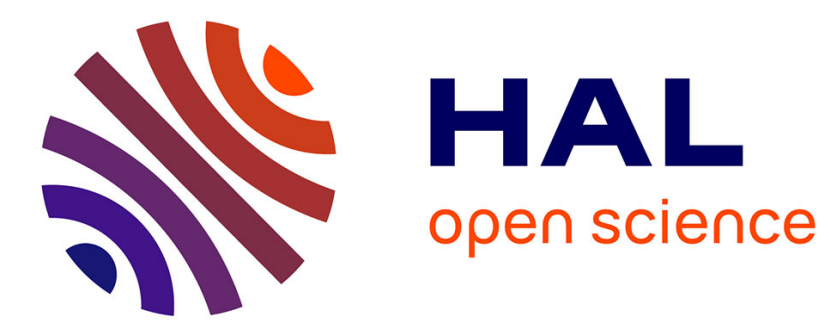

\title{
Passivation de l'InP par oxydation en plasma RF oxygène et par action de HNO3 vapeur
}

\author{
Christine Michel, B. Lepley, B. Bouchikhi, S. Ravelet
}

\section{To cite this version:}

Christine Michel, B. Lepley, B. Bouchikhi, S. Ravelet. Passivation de l'InP par oxydation en plasma RF oxygène et par action de HNO3 vapeur. Revue de Physique Appliquée, 1983, 18 (12), pp.745-749. 10.1051/rphysap:019830018012074500 . jpa-00245141

\section{HAL Id: jpa-00245141 https://hal.science/jpa-00245141}

Submitted on 1 Jan 1983

HAL is a multi-disciplinary open access archive for the deposit and dissemination of scientific research documents, whether they are published or not. The documents may come from teaching and research institutions in France or abroad, or from public or private research centers.
L'archive ouverte pluridisciplinaire HAL, est destinée au dépôt et à la diffusion de documents scientifiques de niveau recherche, publiés ou non, émanant des établissements d'enseignement et de recherche français ou étrangers, des laboratoires publics ou privés. 


\title{
Passivation de l'InP par oxydation en plasma RF oxygène et par action de $\mathrm{HNO}_{3}$ vapeur
}

\author{
C. Michel, B. Lepley, B. Bouchikhi et S. Ravelet \\ Laboratoire d'Electronique et de Physique des Interfaces, I.S.I.N., 54500 Vandœuvre, France
}

(Reçu le 25 juillet 1983, accepté le 13 septembre 1983)

\begin{abstract}
Résumé. - Des oxydes natifs minces ont été élaborés sur la face (100) de (n) InP dans un plasma RF oxygène et dans $\mathrm{HNO}_{3}$ vapeur à des températures allant jusqu'à $200^{\circ} \mathrm{C}$. Les propriétés de ces oxydes ont été étudiées par ellipsométrie et par les méthodes électriques (capacité-tension à $1 \mathrm{MHz}$ et courant-tension) sur les structures métaloxyde-InP. Les épaisseurs d'oxyde atteignent $260 \AA$ avec un indice de réfraction voisin de 2 et une constante diélectrique variant de 4 à 8 .
\end{abstract}

\begin{abstract}
Thin native oxides have been grown on (100) (n) InP in an $\mathrm{O}_{2} \mathrm{RF}$ plasma and in $\mathrm{HNO}_{3}$ gas at different temperatures up to $200^{\circ} \mathrm{C}$. Oxides properties have been studied by ellipsometry and by electrical characterization (capacitance-voltage at $1 \mathrm{MHz}$ and current-voltage) of the metal-oxide-InP structures. Oxide thicknesses get to $260 \AA$ with a refractive index near 2 and a dielectric constant varying from 4 to 8 .
\end{abstract}

\section{Introduction.}

Le phosphure d'indium présente des qualités de surface compatibles avec la réalisation de transistors à effet de champ à grille isolée (TECMIS) [1] mais le dépôt d'isolant ne peut pas se faire à température élevée à cause de la fragilité de la surface : départ de phosphore à partir d'une température de $350^{\circ} \mathrm{C}$ et dislocation du cristal sur plusieurs distances interatomiques par pulvérisation de l'isolant [2].

C'est pourquoi nous avons utilisé deux voies différentes pour faire croître des couches d'oxyde natif d'épaisseurs comprises entre 50 et $200 \AA$ : oxydation dans un plasma $R F$ d'oxygène pur et par action de l'acide nitrique vapeur. Ce sont deux méthodes basse température et qui sont compatibles avec les techniques de fabrication des circuits intégrés. Nous étudions dans cet article la relation entre les méthodes d'oxydation et les propriétés de l'oxyde, déterminées par des mesures optiques et électriques.

\section{Méthodes de préparation des oxydes.}

2.1 Préparation de la SURface. - On utilise soit des couches d'InP épitaxié d'épaisseur 3 à $5 \mu \mathrm{m}$ de densité de donneurs 1 à $9 \times 10^{22} \mathrm{~m}^{-3}$ sur substrat $\mathrm{n}^{+}$provenant du LCR Thomson CSF, soit des échantillons massifs non volontairement dopés de densité $2 \times 10^{22} \mathrm{~m}^{-3}$ provenant du CNET. Les contacts ohmiques sur la face arrière sont réalisés par évaporation de l'eutectique or-germanium recouvert d'or.

Les surfaces $(100)$ de InP sont traitées par voie chimique à l'aide des trois procédés qui donnent les meilleurs résultats de reproductibilité :

a) $\mathrm{HCl} 3 \mathrm{~N}$ pendant $5 \mathrm{~min}$. sur épitaxie,

b) $\mathrm{Br}_{2} 1 \%$ dans $\mathrm{CH}_{3} \mathrm{OH}$ pendant $30 \mathrm{~s}$ ou $2 \mathrm{~min}$. sur le cristal massif,

c) $\mathrm{HF} 40 \%$ pendant $1 \mathrm{~min}$. sur le cristal massif et sur épitaxie.

Les traitements $a$ ) et $c$ ) permettent d'obtenir une surface de bonne qualité : la figure 1 qui représente la photographie de la surface obtenue au microscope à balayage après traitement $\mathrm{HF}$ montre une surface sans rugosité. La photographie 2 montre l'action du brome pendant $2 \mathrm{~min}$. et révèle une surface perturbée. Les acides $\mathrm{HCl}$ et $\mathrm{HF}$ enlèvent l'oxyde naturel alors que le brome attaque en plus l'InP. Le décapage ionique avec des ions argon a été écarté à cause de la fragilité de la surface; on peut observer sur la figure 3 l'effet d'un bombardement par des ions argon de $100 \mathrm{eV}$ pendant $2 \mathrm{~min}$.

2.2 OXYDATION DE LA SURFACE. - Les surfaces décapées sont maintenues dans le propanol 2 ou dans une atmosphère $\mathrm{N}_{2}+\mathrm{H}_{2}$ avant d'être introduits dans le réacteur d'oxydation. 


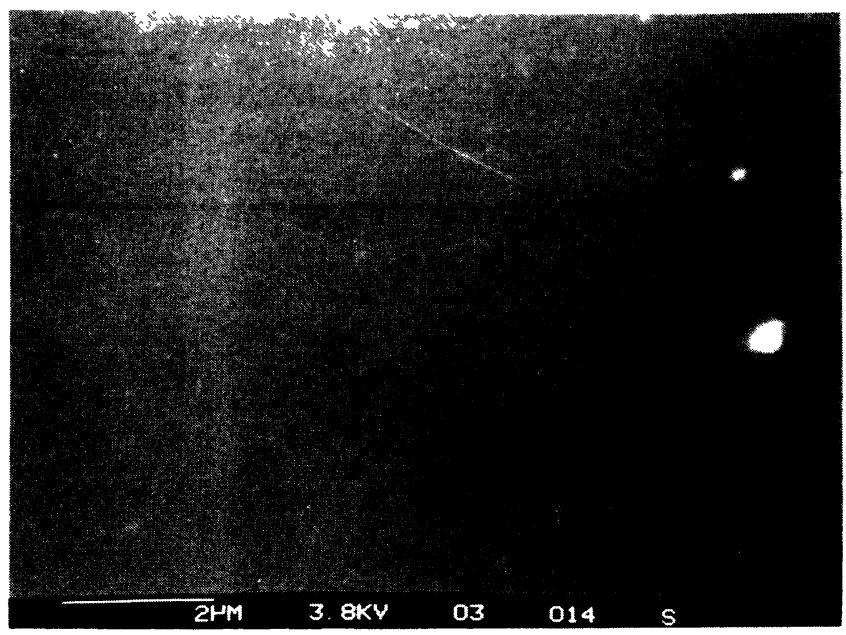

Fig. 1. - Surface d'InP après un traitement HF $40 \% 2$ min. [InP surface after etching with $40 \% \mathrm{HF}$ during $2 \mathrm{~min}$.]

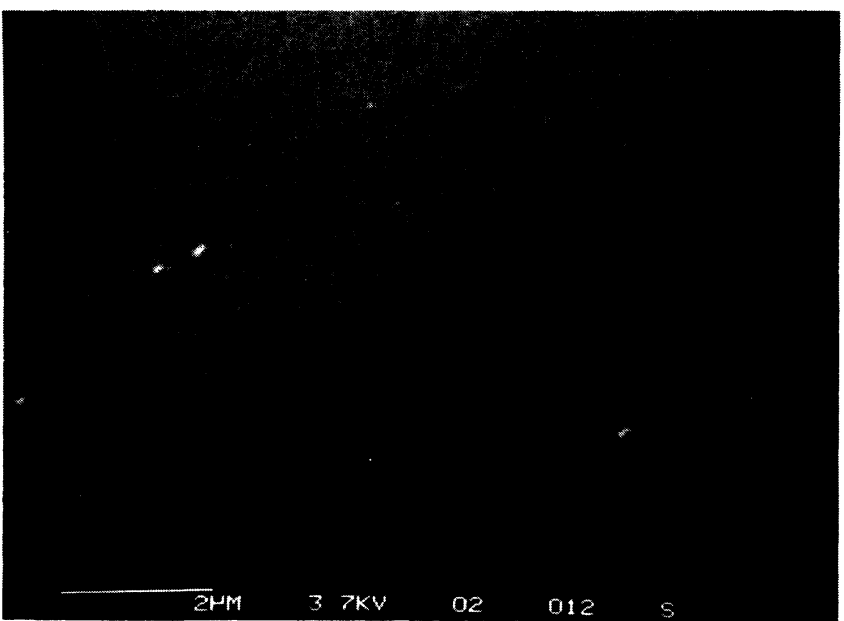

Fig. 2. - Surface d'InP après un traitement $\mathrm{CH}_{3} \mathrm{OH}-\mathrm{Br}_{2}$ $1 \%, 2 \mathrm{~min}$.

[InP surface after etching with $\mathrm{CH}_{3} \mathrm{OH}-\mathrm{Br}_{2} 1 \%$ during $2 \mathrm{~min}$.]

La figure 4 décrit le générateur de plasma $R F$; nous avons travaillé à une pression de $6 \times 10^{-2}$ torr d'oxygène pur pendant 15 et $30 \mathrm{~min}$. avec une puissance RF maximale de $20 \mathrm{~W}$, l'échantillon étant maintenu électriquement isolé dans la décharge sur un support en quartz [3]. Le cristal d'InP peut être chauffé par une lampe quartz-iode placée dans l'enceinte, la température étant repérée par le thermocouple qui passe à l'intérieur du support quartz.

Sur la figure 5 on a représenté le réacteur d'oxydation par acide nitrique vapeur; on a effectué les oxydations à $110^{\circ} \mathrm{C}, 150^{\circ} \mathrm{C}$ et $200^{\circ} \mathrm{C}$ le débit de l'azote d'entraînement étant de $6 \mathrm{~cm}^{3} / \mathrm{min}$.

Les figures 6 et 7 montrent deux états de surface typiques après oxydation. L'oxyde plasma d'épaisseur

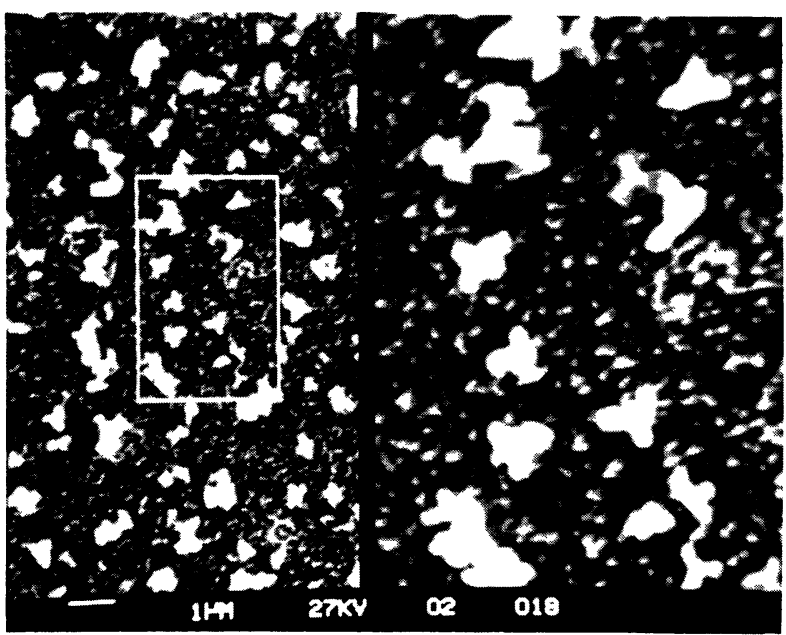

Fig. 3. - Etat de la surface d'InP après bombardement par des ions argon de $100 \mathrm{eV}$.

[InP surface sputtered with $100 \mathrm{eV}$ Ar ions.]

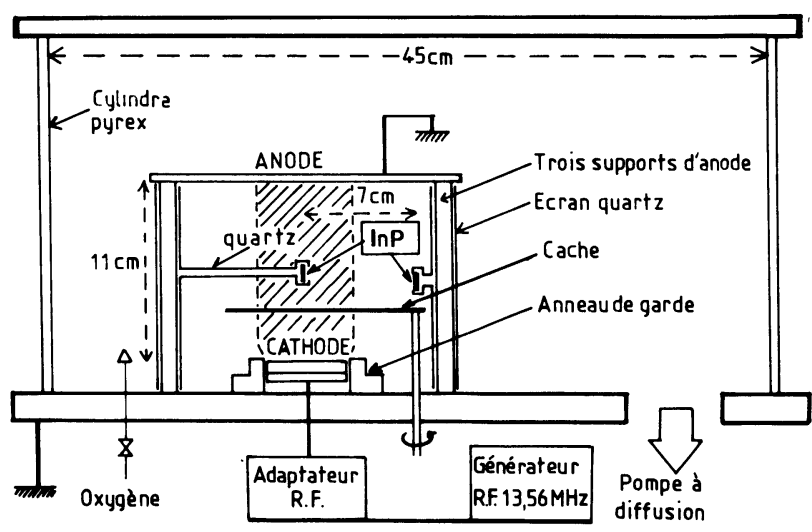

Fig. 4. - Banc d'oxydation Plasma RF.

[RF Plasma-reactor arrangement.]

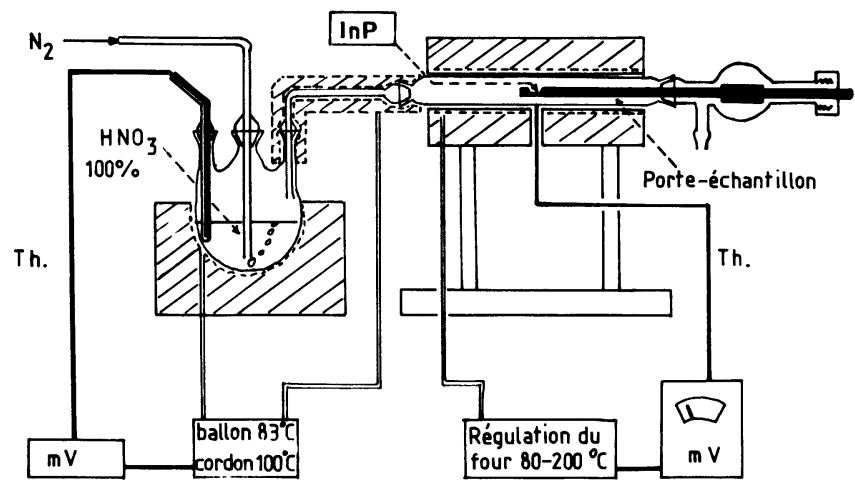

Fig. 5. - Réacteur d'oxydation chimique en phase vapeur. [Apparatus for vapour phase chemical oxidation.] 


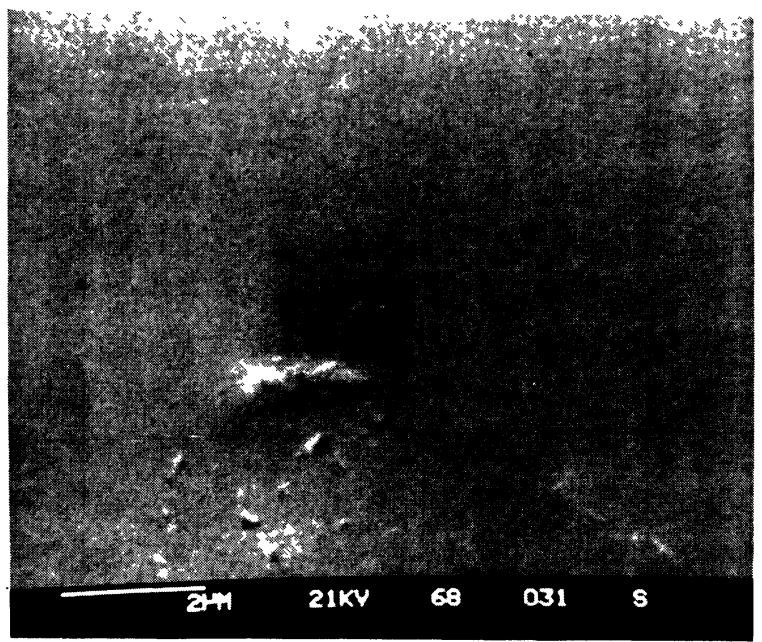

Fig. 6. - Etat de la surface d'InP après oxydation dans un plasma RF. Epaisseur d'oxyde : $50 \AA$.

[InP surface after RF plasma oxidation. Oxide thickness $50 \AA$ Å]

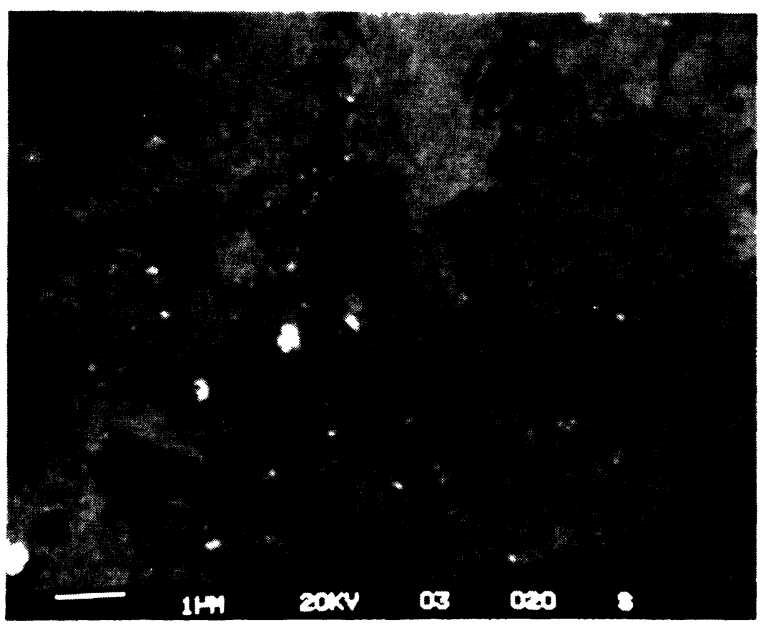

Fig. 7. - Surface d'InP oxydée par $\mathrm{HNO}_{3}$ vapeur. Epaisseur de l'oxyde $130 \AA$.

[InP surface chemically oxidised with $\mathrm{HNO}_{3}$ vapour. Thickness of oxide : $130 \AA$.]

$50 \AA$ (Fig. 6) présente une bonne régularité ainsi qu'on peut le voir autour d'un défaut qui a été choisi pour effectuer la mise au point. La figure 7 montre un aspect d'un oxyde chimique de $130 \AA$ avec une structure présentant un début d'organisation.

\section{Résultats et discussion.}

3.1 EPAISSEUR ET INDICE DES OXYDES. - Les épaisseurs moyennes des oxydes ont été mesurées par ellipsométrie sous une incidence de $70^{\circ}$ à $6328 \AA$ en supposant un indice de l'InP $N=3,548-0,28 i[4,5]$. Les résultats des calculs d'épaisseur et d'indice à partir des paramètres ellipsométriques $\Delta, \psi$ ont été obtenus avec une valeur nulle pour l'indice d'extinction $k_{\text {ox }}$ de l'oxyde, les valeurs non nulles de $k_{\text {ox }}$ conduisant à des épaisseurs incompatibles avec les mesures électriques.

On a représenté sur la figure 8 le paramètre ellipsométrique $\Delta$ en fonction de l'épaisseur $e$ des oxydes.

Pour les faibles épaisseurs (inférieures à $100 \AA$ ) la variation obtenue est linéaire de la forme $\Delta=\Delta_{0}-a e$ et correspond à l'approximation faite pour les faibles différences de marche [6]. Ce résultat est en accord avec les rares résultats publiés sur InP [7].

D'une manière générale on observe que l'épaisseur est toujours inférieure à $100 \AA \AA$ pour un oxyde chimique qui croît sur un substrat massif alors qu'elle dépasse $100 \AA$ dans les mêmes conditions d'oxydation pour une épitaxie. En plasma la différence n'est pas sensible au niveau de l'épaisseur d'oxyde qui est inférieure à $100 \AA$ sauf pour une épitaxie oxydée à $200^{\circ} \mathrm{C}$. Pour l'instant nous n'avons pas d'interprétation de ce phénomène déjà observé par d'autres auteurs [8].

On observe une augmentation de l'épaisseur d'oxyde quand la température de l'InP s'élève ainsi qu'on le voit sur l'encart de la figure 8 pour les oxydes chimiques.

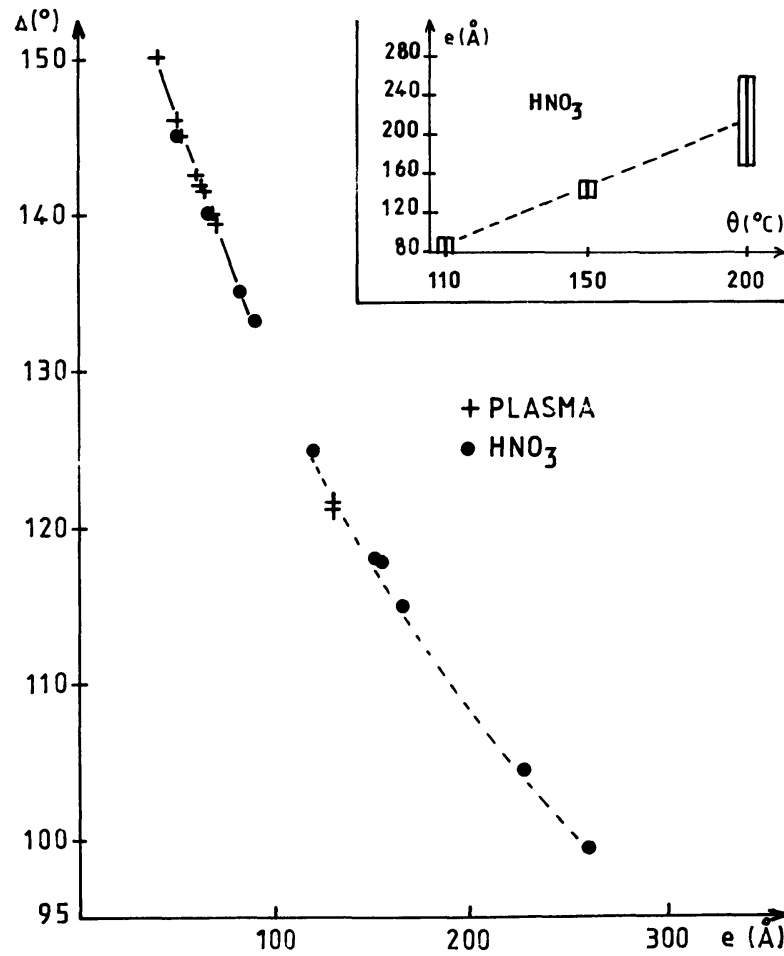

Fig. 8. - Variation du paramètre $\Delta$ en fonction de l'épaisseur des oxydes. Encart : évolution de l'épaisseur avec la température du substrat pour les oxydes préparés avec $\mathrm{HNO}_{3}$ vapeur.

[Variation of the $\Delta$ parameter versus oxide thickness. The insert shows the thickness variation with substrate temperature during $\mathrm{HNO}_{3}$ vapour oxidation.] 
Des paramètres $\Delta, \psi$ on déduit aussi les valeurs des indices $n_{\mathrm{ox}}$ de l'oxyde en fixant $k_{\mathrm{ox}}=0$. Pour les oxydes plasma les valeurs sont dans l'ensemble supérieures à 2 ; elles sont comprises entre 1,9 et 2,4 alors que pour les oxydes chimiques elles sont plus faibles, comprises entre 1,6 et 2,1 mais le plus souvent inférieures à 2 .

Les valeurs élevées des indices pourraient indiquer que l'indice d'extinction n'est pas nul pour l'oxyde plasma ou que l'isolant contient un métal [9]; seules des mesures en ellipsométrie spectroscopique permettront de préciser la composition de l'isolant [10].

3. 2 COMPOSITION DES OXYDES. - Les différences d'indices pour les deux types d'oxydes peuvent être dues à des compositions différentes. Des observations en spectroscopie de photoélectrons X (XPS) [3] ont mis en évidence une composition proche de $\mathrm{InPO}_{4}$ pour l'oxyde plasma alors que l'oxyde chimique contient plus d'oxyde de phosphore.

3. 3 PropriÉtÉs ÉleCtriQues. - Nous ne présentons que les résultats pour des structures élaborées après des préparations de surface à l'aide des acides $\mathrm{HCl}$ et $\mathrm{HF}$ qui donnent une bonne reproductibilité des propriétés électriques.

Afin d'étudier les caractéristiques électriques des oxydes, on réalise des structures MIS par évaporation thermique d'une électrode d'or sur l'oxyde.

Les caractéristiques statiques $I(V)$ ont été utilisées pour déterminer la valeur de la résistivité à faible champ $\left(4 \times 10^{5} \mathrm{~V} \mathrm{~cm}^{-1}\right)$, le champ de claquage et comparer les courants de fuite à travers la structure.

Les résistivités maximales sont de l'ordre de $9 \times 10^{11} \Omega \mathrm{cm}$ et le champ de claquage supérieur à $10^{6} \mathrm{~V} \mathrm{~cm}^{-1}$ pour les deux types d'oxydes.

La valeur minimum des densités de courant à $-1 \mathrm{~V}$ est $10^{-7} \mathrm{~A} \mathrm{~cm}^{-2}$ pour l'oxyde chimique et $5 \times 10^{-7} \mathrm{~A} \mathrm{~cm}^{-2}$ pour l'oxyde plasma.

Les caractéristiques $C(V)$ à $1 \mathrm{MHz}$ ont été tracées à l'aide d'un montage prenant en compte les fortes variations des capacités $(100 \mathrm{pF}$ à $10 \mathrm{nF})$ et des conductances $\left(10^{-6}\right.$ à $\left.10^{-1} \mathrm{~S}\right)$ [11]. La figure 9 montre la caractéristique d'un oxyde plasma obtenu à $200^{\circ} \mathrm{C}$ et d'un oxyde chimique à $150^{\circ} \mathrm{C}$.

De la valeur de la capacité $C_{\text {ox }}$ en accumulation et de l'épaisseur déduite de l'ellipsométrie on tire la constante diélectrique à $1 \mathrm{MHz}$.

a) Oxydation plasma : quel que soit le substrat ou la préparation on obtient une valeur voisine de 5 pour la constante diélectrique des oxydes préparés à température ambiante. Par contre pour l'oxyde préparé à $200^{\circ} \mathrm{C}$ la valeur de $\varepsilon$ atteint 8 ce qui pourrait indiquer une différence de composition ou de structure.

b) Oxydation chimique $\mathrm{HNO}_{3}$ : les valeurs trouvées, comprises entre 4 et 5 , sont plus faibles que pour l'oxyde plasma et correspondent à la valeur 4,5 publiée récemment à propos des oxydes anodiques sur InP [12].

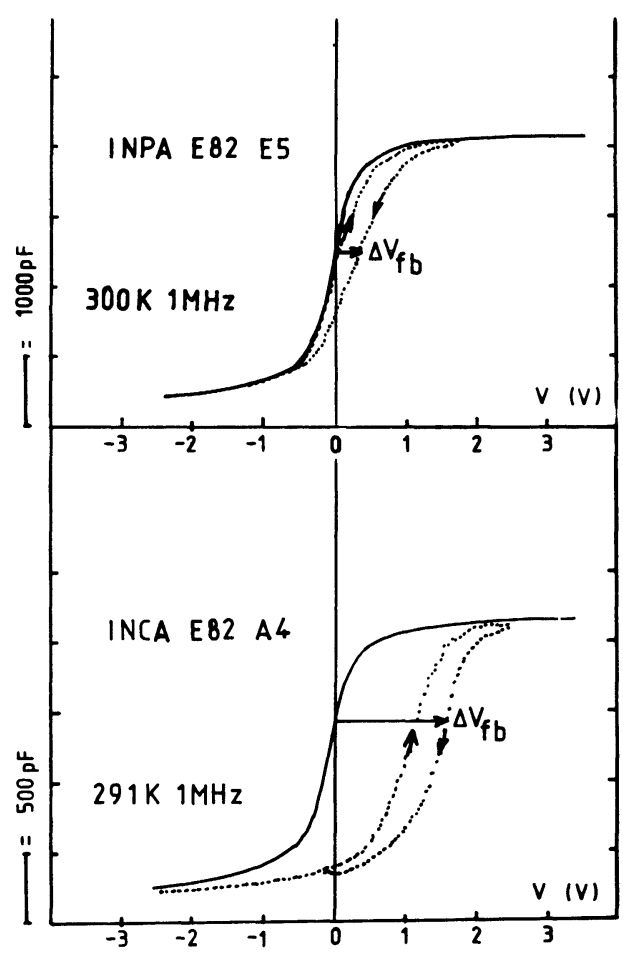

Fig. 9. - Caractéristiques capacité-tension à $1 \mathrm{MHz}$ des structures MIS InP. E5 : oxyde plasma préparé à $200^{\circ} \mathrm{C}$, épaisseur $130 \AA$. A4 : oxyde chimique préparé à $150^{\circ} \mathrm{C}$, épaisseur $160 \AA$.

[1 $\mathrm{MHz}$ capacitance-voltage curves for InP MIS structures. E5 sample : plasma oxide, thickness $130 \AA$, substrate temperature $200^{\circ} \mathrm{C}$. A4 sample : chemical oxide, thickness $160 \AA$, substrate temperature $150^{\circ} \mathrm{C}$.]

Les courbes capacité-tension présentent de l'hystérésis dont l'amplitude augmente avec la tension appliquée et diminue avec la température de mesure; ces deux observations ajoutées au sens de l'hystérésis indiquent une injection d'électrons à l'interface oxydesemiconducteur. Un recuit sous azote pur à $300^{\circ} \mathrm{C}$ permet de diminuer considérablement l'hystérésis.

Dans tous les cas les courbes expérimentales $C(V)$ sont décalées vers les tensions positives par rapport à la courbe calculée; ce décalage est relié à la fois à la différence $\phi_{\mathrm{ms}}$ des travaux de sortie entre métal et semiconducteur et à la densité de charges fixes contenues dans l'oxyde. Les calculs effectués donnent des valeurs comprises entre 0 et $10^{12} \mathrm{~cm}^{-2}$ pour la densité des charges fixes mais la valeur utilisée $\phi_{\mathrm{ms}}=0,4 \mathrm{eV}$ ne correspond pas nécessairement aux conditions expérimentales [13].

\section{Conclusion.}

Les résultats présentés montrent la possibilité de réaliser des oxydes natifs avec des qualités acceptables sur InP. Ces oxydes peuvent être obtenus avec des épaisseurs plus élevées sur un substrat chauffé mais nous nous limiterons à des températures inférieures à $350^{\circ} \mathrm{C}$ pour ne pas détériorer l'interface. Afin 
d'obtenir un isolant d'épaisseur suffisante pour le transistor à effet de champ, un dépôt d'alumine évaporée par un canon à électrons a été réalisé [14] sur l'oxyde natif plasma; des essais sont poursuivis avec pour support l'oxyde natif obtenu par action de l'acide nitrique.

\section{Remerciements.}

Les auteurs souhaitent remercier C. Boutrit et J. M. Guillot pour la réalisation des programmes de calculs ellipsométriques ainsi que N. T. Linh du LCR Thomson CSF qui a fourni les épitaxies élaborées en phase vapeur. Nous remercions aussi G. Post du CNET Bagneux qui a permis une vérification des mesures ellipsométriques et le CNET Lannion pour son support financier (contrat $n^{\circ} 8235010$ ).

Cet article reprend l'exposé oral et tient compte des questions et remarques des participants aux Journées Nationales Semiconducteurs Composés III.V des 4 et 5 juillet 1983 à Toulouse.

\section{Bibliographie}

[1] Meiners, L. G., J. Vac. Sci. Technol. 19 (3) (1981) 373.

[2] Takahashi, Y., Sakai, S., Umeno, M. and Hattori, S., Jpn. J. Appl. Phys. 21 (12) (1982) 1689.

[3] Michel, C., Guillot, J. M., Lepley, B., DupontPavlosky, N. and Karnicka-Moscicka, K., J. Phys. D Appl. Phys. (1983) acceptée 6 juillet 1983.

[4] Whalen, M. S. and Stone, J., J. Appl. Phys. 53 (6) (1982) 4340.

[5] Seraphin, B. O. and Bennet, H. E., Semiconductors and semimetals 3 (New York Academic Press) 1967, chap. 12.

[6] Donaldson, G. B. and Faghihi-Nejad, H., IEEE Trans. Electron. Devices ED 27 (10) (1980) 1988.

[7] Burkhard, H., Dinges, H. W. and Kuphal, E., J. Appl. Phys. 53 (1) (1982) 655.
[8] Clark, D. T., Fok, T., Roberts, G. G. and Sykes, R. W., Thin Solid Films 70 (1980) 261.

[9] Aspnes, D. E., Schwartz, B., Studna, A. A., Derick, L. and Koszi, L. A., J. Appl. Phys. 48 (8) (1977) 3510 .

[10] Theeten, J. B. and Erman, M., J. Vac. Sci. Technol. 20 (3) (1982) 471.

[11] Guillot, J. M., Thèse Docteur-Ingénieur Nancy (1983).

[12] Eftekhari, G., De Cogan, D. and Tuck, B., Phys. Status Solidi (a) 76 (1983) 331.

[13] Nicollian, E. H. and Brews, J. R., MOS Physics and Technology (John Wiley and Sons) 1982, chap. 10.

[14] Blanchet, R., Garrigues, M. et Viktorovitch, P., Congrès INFOS Eindhoven (1983). 